\title{
Set-Valued Stochastic Integrals with Respect to Finite Variation Processes
}

\author{
Jinping Zhang", Jiajia Qi \\ Department of Mathematics and Physics, North China Electric Power University, Beijing, China \\ Email: "zhangjinpingxzy@gmail.com, bycqjj@126.com
}

Received October 31, 2013; revised November 30, 2013; accepted December 6, 2013

Copyright (C) 2013 Jinping Zhang, Jiajia Qi. This is an open access article distributed under the Creative Commons Attribution License, which permits unrestricted use, distribution, and reproduction in any medium, provided the original work is properly cited. In accordance of the Creative Commons Attribution License all Copyrights (C) 2013 are reserved for SCIRP and the owner of the intellectual property Jinping Zhang, Jiajia Qi. All Copyright (C) 2013 are guarded by law and by SCIRP as a guardian.

\begin{abstract}
In a Euclidean space $R^{d}$, the Lebesgue-Stieltjes integral of set-valued stochastic processes $F=\left\{F_{t}(\omega), t \in[0, T]\right\}$ with respect to real valued finite variation process $\left\{A_{t}(\omega), t \in[0, T]\right\}$ is defined directly by employing all integrably bounded selections instead of taking the decomposable closure appearing in some existed references. We shall show that this kind of integral is measurable, continuous in $t$ under the Hausdorff metric and $L^{2}$-bounded.
\end{abstract}

Keywords: Set-Valued Stochastic Process; Finite Variation Process; Measurability

\section{Introduction}

Recently, integrals for set-valued stochastic processes with respect to Brownian motion, martingales and the Lebesgue measure have received much attention.

In 1997, Kisielewicz ([1]) defined the integral of setvalued process as a subset of $L^{2}$ space, but he didn't consider the measurability of the integral. In 1999, Kim and Kim [2] used the definition of stochastic integrals of set-valued stochastic process with respect to the Brownian motion. They called it Aumann ([3]) type It $\hat{o}$ integrals. In [4], Jung and Kim modified the definition by taking the decomposable closure such that the integral is measurable. Li and Ren [5] modified Jung and Kim's definition by considering the predictable set-valued stochastic process as a set-valued random variable in the product space $\left(\mathbb{R}_{+} \times \Omega\right)$, and the measurability and decomposability also were based on product $\sigma$-algebra. After that, Zhang et al. ([6,7]) studied the set-valued integrals with respect to the martingale and Brownian motion.

Stochastic differential inclusions and set-valued stochastic differential (or integral) equations are employed to model the problems with not only randomness but also

${ }^{*}$ This work is partly supported by The Co-Construction Project of Beijing Municipal Commission of Education, The Project Sponsored by SRF for ROCS, SEM and The Fundamental Research Funds for the Central Universities, No 12MS81.

${ }^{\#}$ Corresponding author. impreciseness. Recently, there are some references related to set-valued differential equations such as [8-13] etc.

Concerning to the integral with respect to finite variation processes, Malinowski and Michta [12] give the notion of set-valued integral with respect to single valued finite variation but without considering the measurability. Z.Wang and R.Wang [14] defined the Lebesgue-Stieltjes stochastic integral of single valued stochastic processes with respect to set-valued finite variation processes (refer to [14] for the detail).

In this paper, different from the definition in [14], based on the Definition 3.1 in [12], we will study the Lebesgue-Stieltjes integral of set-valued stochastic processes with respect to single valued finite variation process. We shall prove the measurability of integral, namely, it is a set-valued random, which is similar to the classical stochastic integral.

This paper is organized as follows: in Section 2, we present some notions and facts on set-valued random variables; in Section 3, we shall give the definition of integral of set-valued stochastic processes with respect to finite variation process and then prove the measurability and $L^{2}$-boundedness.

\section{Preliminaries}

We denote $\mathbb{N}$ the set of all natural numbers, $R$ the set 
of all real numbers, $R^{d}$ the d-dimensional Euclidean space with the usual norm $\|\cdot\|, R_{+}$the set of all nonnegative numbers. Let $(\Omega, \mathcal{F}, P)$ be a complete probability space, $\left\{\mathcal{F}_{t}: t \in[0, T]\right\}$ a $\sigma$-field filtration satisfying the usual conditions. Let $\mathcal{B}(E)$ be a Borel field of a topological space $E$.

Let $\mathcal{K}\left(R^{d}\right)$ (resp. $\mathcal{K}_{k}\left(R^{d}\right), \mathcal{K}_{k c}\left(R^{d}\right)$ ) be the family of all nonempty, closed (resp. nonempty compact, nonempty compact convex) subsets of $R^{d}$. For any $x \in R^{d}$ and $A \in \mathcal{K}\left(R^{d}\right)$, define the distance between $x$ and $A$ by $d(x, A)=\inf _{y \in A}\|x-y\|$. The Hausdorff metric $d_{H}$ on $\mathcal{K}\left(\mathcal{R}^{d}\right)$ (see e.g. [15]) is defined by

$$
d_{H}(A, B)=\max \left\{\sup _{a \in A} d(a, B), \sup _{b \in B} d(b, A)\right\}
$$

$A, B \in \mathcal{K}\left(R^{d}\right)$.

Denote $\|A\|_{k}=d_{H}(\{0\}, A)=\sup _{a \in A}\|a\|$. For

$A, B, C, D \in \mathcal{K}^{(}\left(R^{d}\right)$, we have

$$
H(A+B, C+D) \leq H(A, C)+H(B, D) .
$$

For $A \subset R^{d}, x^{*} \in R^{d}$ the support function of $A$ is defined as follows:

$$
S\left(x^{*}, A\right)=\sup \left\{\left\langle x^{*}, x\right\rangle: x \in A\right\} .
$$

$L^{p}\left(\Omega, \mathcal{F}, P ; R^{d}\right)=L^{p}\left(\Omega ; R^{d}\right)(p \geq 1)$ : the set of all $R^{d}$-valued Borel measurable functions $f: \Omega \rightarrow R^{d}$ such that the norm

$$
\begin{aligned}
& \|f\|_{p}=\left\{\int_{\Omega}\|f(\omega)\|^{p} \mathrm{~d} P\right\}^{\frac{1}{p}}, \text { if } 1 \leq p<\infty, \\
& \|f\|_{\infty}=\operatorname{essup}_{\omega \in \Omega}\|f(\omega)\|, \text { if } p=\infty,
\end{aligned}
$$

is finite. $f$ is called $L^{p}$-integrable if $f \in L^{p}\left(\Omega ; R^{d}\right)$.

A set-valued function $F: \Omega \rightarrow \mathcal{K}\left(R^{d}\right)$ is said to be measurable if for any open set $O \subset R^{d}$, the inverse $F^{-1}(O):=\{\omega \in \Omega: F(\omega) \bigcap O \neq \varnothing\}$ belongs to $\mathcal{F}$. Such a function $F$ is called a set-valued random variable.

Let $\mathcal{M}\left(\Omega, \mathcal{F}, P ; \mathcal{K}\left(R^{d}\right)\right)$ (resp. $\mathcal{M}\left(\Omega, \mathcal{F}, P ; \mathcal{K}_{c}\left(R^{d}\right)\right)$, $\mathcal{M}\left(\Omega, \mathcal{F}, P ; \mathcal{K}_{k c}\left(R^{d}\right)\right)$ ) be the family of all measurable $\mathcal{K}\left(R^{d}\right)$-valued (resp. $\mathcal{K}_{c}\left(R^{d}\right), \mathcal{K}_{k c}\left(R^{d}\right)$-valued) functions, briefly by $\mathcal{M}\left(\Omega ; \mathcal{K}\left(R^{d}\right)\right)$ (resp. $\mathcal{M}\left(\Omega, \mathcal{F}, P ; \mathcal{K}_{c}\left(R^{d}\right)\right), \mathcal{M}\left(\Omega, \mathcal{F}, P ; \mathcal{K}_{\text {kc }}\left(R^{d}\right)\right)$.

For $F \in \mathcal{M}\left(\Omega, K\left(R^{d}\right)\right)$, the family of all $L^{p}$-integrable selections is defined by

$$
\begin{aligned}
& S_{F}^{p}(\mathcal{F}):=\left\{f \in L^{p}\left(\Omega, \mathcal{F}, P ; R^{d}\right): f(\omega) \in F(\omega) \text { a.s. }\right\}, \\
& p \geq 1
\end{aligned}
$$

In the following, $S_{F}^{p}(\mathcal{F})$ is denoted briefly by $S_{F}^{p}$.

A set-valued random variable $F$ is said to be integrable if $S_{F}^{1}$ is nonempty. $F$ is called $L^{p}(p \geq 1)$-integrably bounded if there exits $h \in L^{p}\left(\Omega, \mathcal{F}, P ; R^{d}\right)$ s.t. for all $x \in F(\omega),\|x\| \leq h(\omega)$ almost surely.

An $R^{d}$-valued stochastic process $f=\left\{f_{t}: t \geq 0\right\}$ (or denoted by $f=\{f(t): t \geq 0\}$ ) is defined as a function $f: R_{+} \times \Omega \rightarrow R^{d}$ with the $\mathcal{F}$-measurable section $f_{t}$, for $t \geq 0$. We say $f$ is measurable if $f$ is $\mathcal{B}_{+} \otimes \mathcal{F}$ measurable. The process $f=\left\{f_{t}: t \geq 0\right\}$ is called $\mathcal{F}_{t}$-adapted if $f_{t}$ is $\mathcal{F}_{t}$-measurable for every $t \geq 0$. Let $\Sigma:=\bigcap_{t \geq 0}\left\{Z \in \mathcal{B}\left(R_{+}\right) \otimes \mathcal{F}: Z_{t} \in \mathcal{F}_{t}\right\}$, where $Z_{t}=\{\omega ;(t, \omega) \in Z\}$. We know that $\Sigma$ is a $\sigma$-algebra on $R_{+} \times \Omega$. A function $f: R_{+} \times \Omega \rightarrow R^{d}$ is measurable and $\mathcal{F}_{t}$-adapted if and only if it is $\Sigma$-measurable ([8]).

In a fashion similar to the $R^{d}$-valued stochastic processes, a set-valued stochastic process $F=\left\{F_{t}: t \geq 0\right\}$ is defined as a set-valued function $F: R_{+} \times \Omega \rightarrow \mathcal{K}\left(R^{d}\right)$ with $\mathcal{F}$-measurable section $F_{t}$ for $t \geq 0$. It is called measurable if it is $\mathcal{B}_{+} \otimes \mathcal{F}$-measurable, and $\mathcal{F}_{t}$ adapted if for any fixed $t, F_{t}(\cdot)$ is $\mathcal{F}_{t}$-measurable. $F=\left\{F_{t}: t \geq 0\right\}$ is measurable and $\mathcal{F}_{t}$-adapted if and only if it is $\Sigma$-measurable. $F=\left\{F_{t}: t \geq 0\right\}$ is called $L^{p}$-integrable if every $F_{t}$ is $L^{p}$-integrable.

\section{Set-Valued Stochastic Integral w.r.t Finite Variation Processes}

Let $A=\left\{A_{t}, t \geq 0\right\}$ be a real valued $\mathcal{F}_{t}$-adapted measurable process with finite variation and continuous sample trajectories a.s. from the origin. That is to say, for each compact interval $[s, t] \subset[0, \infty)$ and any partition $\Delta=\left\{t_{1}, \cdots, t_{n}\right\}$ of $[s, t]$, the total variation

$$
V_{A}([s, t])=\sup _{\Delta} \Sigma_{2=1}^{n}\left|A_{t_{i}}(\omega)-A_{t_{i-1}}(\omega)\right|
$$

is finite and $A(0, \cdot)=0$ a.s. Then for any $T>0$, the process $A=\left\{A_{t}, t \geq 0\right\}$ can generate a random measure denoted by $\mu_{A}$ in the space $([0, T],(\mathcal{B}[0, T]))$. For any $(s, t] \subset[0, T]$, let

$$
\mu_{A}((s, t]):=|A(t, \omega)|-|A(s, \omega)|
$$

where $A(t, \omega)=A^{+}(t, \omega)+A^{-}(t, \omega)$ is the decomposition of $A, A^{+}$and $A^{-}$are non-negative and nondecreasing processes, $|A(t, \omega)|=A^{+}(t, \omega)+A^{-}(t, \omega)$. In the product space $(\Omega \times[0, T], \Sigma)$, set

$$
v_{A}(C):=\int_{\Omega} \int_{[0, T]} \mathbf{1}_{C}(t, \omega) \mu_{A}([0, T]) \mu_{A}(\mathrm{~d} t) P(\mathrm{~d} \omega),
$$

for $C \in \Sigma$, where $\mathbf{1}_{C}$ is the index function. Then the set function $v$ is a finite measure in the measurable space $(\Omega \times[0, T], \Sigma)$ if and only if

$\int_{\Omega}\left(\mu_{A}([0, T])\right)^{2} P(\mathrm{~d} \omega)<\infty$. In the following we always

assume $\int_{\Omega}\left(\mu_{A}([0, T])\right)^{2} P(\mathrm{~d} \omega)<\infty$.

Let $L^{2}\left(\Omega \times[0, T], \Sigma, v_{A} ; R^{d}\right)$ be the family of all $\Sigma$-measurable $R^{d}$-valued stochastic processes $f$ such that

$$
\int_{\Omega \times[0, T]}\|f(\omega, t)\|^{2} v_{A}(\mathrm{~d} t)<\infty .
$$


For any $f \in L^{2}\left(\Omega \times[0, T], \Sigma, v_{A} ; R^{d}\right)$ and $[s, t] \subset[0, T]$, the stochastic Lebesgue-Stieltjes integral $\int_{[s, t]} f(\tau) \mathrm{d} A_{\tau}$ is defined by the Bochner integral $\int_{[s, t]} f(\tau)^{[s, t]} \mu_{A}(\mathrm{~d} \tau)$ pathby-path. One can show that the integral process $\left\{\int_{[0, t]} f(s) \mathrm{d} A_{s}, t \in[0, T]\right\}$ is $\Sigma$-measurable.

Note: in [12], the integrand is assumed being predictable, in fact the integrand can be relaxed to the $\Sigma$ measurable class since the integrator $A_{t}$ is continuous.

Let $M^{2}\left(\Omega \times[0, T], \Sigma, v_{A} ; \mathcal{K}\left(R^{d}\right)\right)$ be the family of all $\Sigma$-measurable $\mathcal{K}\left(R^{d}\right)$-valued stochastic processes $F$ such that

$$
\int_{\Omega \times[0, T]}\|F(\omega, t)\|_{k}^{2} v_{A}(\mathrm{~d} t)<\infty,
$$

where $\|F(\omega, t)\|_{k}=\sup _{x \in F(\omega, t)}\|x\|$. For any

$$
\begin{aligned}
& F \in M^{2}\left(\Omega \times[0, T], \Sigma, v_{A} ; \mathcal{K}\left(R^{d}\right)\right) \text {, set } \\
& S^{2}(F):=\left\{f \in M^{2}\left(\Omega \times[0, T], \Sigma, v_{A} ; R^{d}\right):\right. \\
& \left.f(\omega, t) \in F(\omega, t), v_{A}-a . e .\right\}
\end{aligned}
$$

Definition 1. (see [12]) For a set-valued stochastic process $F \in M^{2}\left(\Omega \times[0, T] ; \mathcal{K}_{k c}\left(R^{d}\right)\right)$ the set-valued stochastic Lebesgue-Stieltjes integral (over interval $[s, t]$ ) of $F$ with respect to the finite variation continuous process $A$ is the set

$$
\int_{[s, t]} F(\tau) \mathrm{d} A_{\tau}:=\left\{\int_{[s, t]} f(\tau) \mathrm{d} A_{\tau}: f \in S^{2}(F)\right\} .
$$

In [12], the authors call this kind of integral as trajectory integral since they consider it as a

$\mathcal{K}\left(L^{2}\left(\Omega \times[0, T], \Sigma, v_{A} ; R^{d}\right)\right)$-valued random variable. Here, we shall consider it as a subset of $R^{d}$ and show the measurability with respect to $\mathcal{F}$, which is very different from the way in [12], also different from other references such as $[10,16,17]$ etc. In fact, for almost every $\omega \in \Omega$, the above integral $\int_{[s, t]} F(\tau) \mathrm{d} A_{\tau}$ is a subset of $R^{d}$. In the following, we shall assume the $\sigma$ algebra $\mathcal{F}$ is separable w.r.t $P$. In addition, $\mathcal{B}([0, T])$ is separable and $\Sigma \subset \mathcal{F} \otimes \mathcal{B}([0, T])$, then one can get $S^{2}(F)$ is separable. Therefore we can find an $\mathcal{F}$ measurable set $\Omega_{F}$, such that $P\left(\Omega_{F}\right)=1$ and for every $\omega \in \Omega_{F}$, the integral $\int_{[0, T]} F(\tau) \mathrm{d} A_{\tau}$ is defined path-bypath. For $\omega \in \Omega / \Omega_{F}$, set $\int_{[s, t]} F(\tau) \mathrm{d} A_{\tau}=\{0\}$, therefore it is well defined for every $\stackrel{[s, t]}{\omega \in} \Omega$.

$\int_{(s, t]} F(\tau) \mathrm{d} A_{\tau}=\int_{[s, t]} F(\tau) \mathrm{d} A_{\tau}$ since the continuity of $A_{t}$. In the sequel, we shall denote the integral by $\int_{s}^{t} F(\tau) \mathrm{d} A_{\tau}$ instead of $\int_{[s, t]} F(\tau) \mathrm{d} A_{\tau}$. For any $t \in[0, T]$, denote $\int_{0}^{t} F_{s} \mathrm{~d} A_{s}$ by $I_{t}(F)$.

Theorem 1. For $F \in M^{2}\left(\Omega \times[0, T], \Sigma, \mathcal{K}_{k c}\left(R^{d}\right) ; v_{A}\right)$, $[s, t] \subset[0, T]$ and $\omega \in \Omega$, the Lebesgue-Stieltjes integral $\int_{s}^{t} F_{\tau}(\omega) \mathrm{d} A_{\tau}(\omega)$ is a compact and convex subset of $R^{d}$.

Proof 1. In fact, $S^{2}(F)$ is a bounded and convex subset of $L^{2}\left(\Omega \times[0, T], \Sigma, R^{d} ; v_{A}\right)$, since $F$ is convex and compact,moreover, it is weakly compact since $L^{2}\left(\Omega \times[0, T], \Sigma, R^{d} ; v_{A}\right)$ is reflexive. The convexity of the integral is obvious.

We shall show the linear operator

$\mathcal{T}(F):=\int_{s}^{t} F_{\tau}(\omega) \mathrm{d} A_{\tau}(\omega): S^{2}(F) \rightarrow \mathcal{K}_{k c}\left(R^{d}\right)$ is bounded.

For any $f \in S^{2}(F), \quad[s, t] \subset[0, T]$,

$$
\begin{aligned}
& \left\|\int_{S}^{t} f(\tau, \omega) \mathrm{d} A_{\tau}(\omega)\right\| \leq \int_{s}^{t}\|f(\tau, \omega)\|\left|\mathrm{d} A_{\tau}(\omega)\right| \\
& \leq \int_{s}^{t}\|F(\tau, \omega)\|_{\mathcal{K}}\left|\mathrm{d} A_{\tau}(\omega)\right|<\infty,
\end{aligned}
$$

which implies the linear operator $\mathcal{T}$ is bounded. Therefore the integral $\int_{s}^{t} F_{\tau} \mathrm{d} A_{\tau}$ is weakly compact since the bounded linear operator mapping a weakly compact set to a weakly compact one. In $R^{d}$ space, a weakly compact set is compact.

Lemma 1. (see [16] Corollary 2.1.1 (5)) Assume $(\Omega, \mathcal{A})$ is a measurable space, $\mathfrak{X}$ is a separable Banach space, $F: \Omega \rightarrow \mathcal{K}(\mathfrak{X})$, and $F$ is a set-valued random variable, then $S\left(x^{*}, F(\omega)\right)\left(x^{*} \in \mathfrak{X}^{*}\right)$ is measurable.

By using Lemma 1, as a manner similar to Theorem 1 in [17], we have the following result:

Lemma 2. Assume $A$ is the corresponding stochastic process, $F \in M^{2}\left(\Omega \times[0, T], \Sigma, \mathcal{K}_{k c}\left(R^{d}\right) ; v_{A}\right), \quad$ for any $[s, t] \subset[0, T]$, we have

1) $\int_{s}^{t} \alpha F_{\tau} \mathrm{d} A_{\tau}=\alpha \int_{s}^{t} F_{\tau} \mathrm{d} A_{\tau}, \alpha \in R$;

$$
\text { 2) } S\left(x^{*}, \int_{s}^{t} F_{\tau} \mathrm{d} A_{\tau}\right)=\int_{s}^{t} S\left(x^{*}, F_{\tau}\right) \mathrm{d} A_{\tau}, x^{*} \in R^{d} \text {. }
$$

Lemma 3. (see [16] Theorem 2.1.16) Assume $(\Omega, \mathcal{F})$ is a measurable space, $\mathfrak{X}$ is a separable Banach space, $F: \Omega \rightarrow \mathcal{K}_{k c}(\mathfrak{X})$, and for any fixed $x^{*} \in \mathfrak{X}^{*}, \quad S\left(x^{*}, F\right)$ is measurable, if one of the following conditions is satisfied:

1) $\mathfrak{X}^{*}$ is separable;

2) for any $\omega \in \Omega, F(\omega) \in \mathcal{K}_{k c}(\mathfrak{X})$.

Then $F$ is a set-valued random variable.

From Lemma 1 and Lemma 3, when $\mathfrak{X}=R^{d}$, for any $x^{*} \in R^{d}, \quad F(\omega) \in K_{k c}\left(R^{d}\right)$ is $\mathcal{F}$-measurable if and only if $S\left(x^{*}, F\right)(\omega)$ is $\mathcal{F}$-measurable.

Lemma 4. ([16] Theorem 1.7.7) If $(\Omega, \mathcal{F})$ is a separable space, $\mathfrak{X}, \mathcal{Y}$ are separable metric space $F: \Omega \times \mathfrak{X} \rightarrow \mathcal{K}(\mathcal{Y})$ satisfy:

(a) for any $x \in \mathfrak{X}, \omega \rightarrow F(\omega, x)$ is measurable;

(b) for any $\omega \in \Omega, x \rightarrow F(\omega, x)$ is continuous or is continuous with respect to Hausdorff metric,

Then $(\omega, x) \rightarrow F(\omega, x)$ is jointly measurable.

Then by Lemma 1 we have the following: 
Lemma 5. Assume $F \in M^{2}\left([0, T] \times \Omega, \Sigma, \mathcal{K}_{k c}\left(R^{d}\right) ; v_{A}\right)$. Then $S\left(x^{*}, F(t, \omega)\right):[0, T] \times \Omega \rightarrow R$ is $\Sigma$-measurable.

Theorem 2. Assume

$F \in M^{2}\left([0, T] \times \Omega, \Sigma, \mathcal{K}_{k c}\left(R^{d}\right) ; v_{A}\right)$. Then

$I_{t}(F) \in L^{2}\left(\Omega ; R^{d}\right)$ for each $t \in[0, T]$. Furthermore, the mapping $\psi(t, \omega)=I_{t}(F)$ is $\left(\mathcal{B}[0, t] \otimes \mathcal{F}_{t}\right)$-measurable.

Proof 2. Step 1. We will show that $I_{t}(F)$ is $\mathcal{F}_{t}$ measurable for each $t \in[0, T], \psi(t, \omega)=I_{t}(F)$ is $\left(\mathcal{B}[0, T] \times \mathcal{F}_{T}\right)$-measurable.

By Theorem 1, we have

$$
\begin{aligned}
& I_{T}(F)(\omega)=\int_{0}^{T} F_{t}(\omega) \mathrm{d} A_{t}(\omega) \\
& =\left\{\int_{0}^{T} f_{t}(\omega) \mathrm{d} A_{t}(\omega) ; f \in S^{2}(F)\right\} \in \mathcal{K}_{k c}\left(R^{d}\right)
\end{aligned}
$$

for all $\omega \in \Omega$. Furthermore, we obtain

$$
S\left(x^{*}, I_{t}(F)(\omega)\right)=\int_{0}^{t} S\left(x^{*}, F(s, \omega)\right) \mathrm{d} A_{s}
$$

for all $\omega \in R^{d}$. Moreover, since

$F(t, \omega):[0, t] \times \Omega \rightarrow \mathcal{K}_{k}\left(R^{d}\right)$ is

$\left(\mathcal{B}[0, t] \otimes \mathcal{F}_{t}\right)$-measurable, from the Lemma 5 we can obtain that the function $S\left(x^{*}, F(s, \omega)\right):[0, T] \times \Omega \rightarrow R$ is $\left(\mathcal{B}([0, T]) \otimes \mathcal{F}_{T}\right)$ measurable. By Fubini theorem, $\int_{0}^{t} S\left(X^{*}, F(s, \omega)\right) \mathrm{d} A_{s}$ is $\mathcal{F}_{t}$-measurable, based on Lemma $3, I_{t}(F)$ is $\mathcal{F}_{T}$-measurable.

Finally, in the argument above, the function $\phi(t, \omega)=\int_{0}^{t} S\left(x^{*}, F(s, \omega)\right) \mathrm{d} A_{s}$ is $\mathcal{F}_{T}$-measurable for each $t \in[0, T]$. Since it is continuous in $t \in[0, T]$ for all $\omega \in \Omega$, so it is $\left(\mathcal{B}([0, T]) \otimes \mathcal{F}_{T}\right)$-measurable. From Lemma 4 , we obtain that $I_{t}(F)(\omega)$ is $\left(\mathcal{B}([0, t]) \otimes \mathcal{F}_{t}\right)$ measurable.

Step 2. In this step, we will show that

$I_{t}(F)(\omega) \in L^{2}\left(\Omega ; \mathcal{K}\left(R^{d}\right)\right)$ for each $t \in[0, T]$. have

For each $\omega \in \Omega, t \in[0, T]$ and $f \in S^{2}(F)(\omega)$, we

$$
\begin{aligned}
\left\|\int_{0}^{t} f_{s} \mathrm{~d} A_{s}\right\|^{2} & \leq \mu_{A}([0, T]) \int_{0}^{t}|| f_{s} \|^{2}\left|\mathrm{~d} A_{s}\right| \\
& \leq \mu_{A}([0, T]) \int_{0}^{t}|| F_{s} \|_{k}^{2}\left|\mathrm{~d} A_{s}\right|
\end{aligned}
$$

then

$$
\sup _{f \in S^{2}(F)}\left\|\left|\int_{0}^{t} f_{s} \mathrm{~d} A_{s}\left\|^{2} \leq \mu_{A}([0, T]) \int_{0}^{t}\right\| F_{s} \|_{k}^{2}\right| \mathrm{d} A_{s} \mid\right.
$$

Hence,

$$
\begin{aligned}
& E\left[\left\|I_{t}(F)(\omega)\right\|_{k}^{2}\right] \\
\leq & E\left[\int_{0}^{T} \mu_{A}([0, T])\|F(t, \omega)\|_{k}^{2}\left|\mathrm{~d} A_{s}\right|\right] \\
\leq & \int_{\Omega \times[0, T]}\left\|F_{s}\right\|_{k}^{2} v_{A}(\mathrm{~d} s)<\infty,
\end{aligned}
$$

which implies

$$
I_{t}(F) \in L^{2}\left(\Omega ; \mathcal{K}\left(R^{d}\right)\right) .
$$

As a manner similar to Theorem 3.8. in [8], we have the Castaing representation as following:

Theorem 3. For a set-valued stochastic process $F \in M^{2}\left([0, T] \times \Omega ; \mathcal{K}_{k c}\left(R^{d}\right), v_{A}\right)$, there exists a sequence $\left\{f^{n} ; n \in \mathbb{N}\right\} \subset S^{2}(F)$ such that

$$
F_{t}(\omega)=\operatorname{cl}\left\{f_{t}^{n}(\omega) ; n \in \mathbb{N}\right\} \text { for a.e. }(t, \omega),
$$

and, for $0 \leq s \leq t \leq T$,

$$
\left.I_{s, t}(F)(\omega)=\operatorname{cl}\left\{\int_{s}^{t} f_{u}^{n}(\omega) \mathrm{d} A_{u} ; n \in \mathbb{N}\right\}\right\} \text { a.s, }
$$

where cl denotes the closure in $R^{d}$.

Theorem 4. For each $F \in M^{2}\left([0, T] \times \Omega ; \mathcal{K}_{k c}\left(R^{d}\right), v_{A}\right)$, $I_{t}(F)(\omega)$ is continuous a.s. with respect to the Hausdorff metric $d_{H}$.

Proof 3. Let $0 \leq r<t \leq T$ and $\omega \in \Omega$. We then have

$$
\begin{aligned}
& I_{T}\left(\mathbf{1}_{[0, t]} F\right)(\omega)=I_{T}\left(\mathbf{1}_{[0, r]} F+\mathbf{1}_{[r, t]} F\right)(\omega) \\
& =I_{T}\left(\mathbf{1}_{[0, r]} F\right)(\omega)+I_{T}\left(\mathbf{1}_{[r, t]} F\right)(\omega)
\end{aligned}
$$

Hence,

$$
\begin{aligned}
& d_{H}\left(I_{t}(F)(\omega), I_{r}(F)(\omega)\right) \\
& =d_{H}\left(I_{T}\left(\mathbf{1}_{[0, r]} F\right)(\omega)+I_{T}\left(\mathbf{1}_{[r, t]} F\right)(\omega), I_{T}\left(\mathbf{1}_{[0, r]}(F)(\omega)\right)\right) \\
& \leq d_{H}\left(I_{T}\left(\mathbf{1}_{[r, t]} F(\omega),\{0\}\right)\right) \\
& =\sup _{f \in S_{T}(F)(\omega)}\left\|\int_{0}^{t} f(s) \mathrm{d} A_{s}\right\| \\
& \leq \int_{r}^{t}\|F(s, \omega)\|_{k}\left|\mathrm{~d} A_{s}\right|<\infty
\end{aligned}
$$

since for each $f \in S_{T}(F)(\omega)$,

$\left\|\int_{0}^{t} f(s) \mathrm{d} A_{s}\right\| \leq \int_{r}^{t}\|F(s, \omega)\|_{\mathcal{K}}\left|\mathrm{d} A_{s}\right|$. Hence,

$\lim _{r \uparrow t} \int_{r}^{t}\|F(s, \omega)\|_{\mathcal{K}} \mathrm{d} A_{s}(\omega)=0$. So $I_{t}(F)(\omega)$ is leftcontinuous in $t \in[0, T]$ for all a.s. In a similar way, we see that $I_{t}(F)(\omega)$ is right-continuous in $t \in[0, T]$ a.s.

Similar to the proof of Theorem 3.15 in [8], we have the following theorem:

Theorem 5. Let $F, G \in M^{2}\left(\Omega \times[0, T], \Sigma, v_{A} ; \mathcal{K}_{k c}\left(R^{d}\right)\right)$, for any $t \in[0, T]$, we have

$$
E\left[d_{H}\left(I_{t}(F), I_{t}(G)\right)\right] \leq \int_{\Omega \times[0, T]} d_{H}\left(F_{s}, G_{s}\right) v_{A}(\mathrm{~d} s)
$$

and

$$
E\left[d_{H}^{2}\left(I_{t}(F), I_{t}(G)\right)\right] \leq 2 \int_{\Omega \times[0, T]} d_{H}^{2}\left(F_{s}, G_{s}\right) v_{A}(\mathrm{~d} s) .
$$




\section{Conclusion}

When the integrand takes values in compact and convex subsets of $R^{d}$, we defined the integral with respect to real-valued variation processes. And then we proved some properties of this kind of integral such as measurability, $L^{2}$-boundedness and continuity under the Hausdorff metric.

\section{Acknowledgements}

We would like to thank the referees for their valuable comments. Moreover, we express special thanks to our editor of the journal APM for his(her) efficiency and support.

\section{REFERENCES}

[1] M. Kisielewicz, "Set-Valued Stochastic Integrals and Stochastic Inclusions,” Discussiones Mathematicae, Vol. 13, 1993, pp. 119-126.

[2] B. K. Kim and J. H. Kim, "Stochastic Integrals of Set-Valued Processes and Fuzzy Processes," Journal of Mathematical Analysis and Applications, Vol. 236, No. 2, 1999, pp. 480-502.

http://dx.doi.org/10.1006/jmaa.1999.6461

[3] R. J. Aumann, "Intgrals of Set-Valued Functions," Journal of Mathematical Analysis Applications, Vol. 12, No. 1, 1965, pp. 1-12.

http://dx.doi.org/10.1016/0022-247X(65)90049-1

[4] E. J. Jung and J. H. Kim, “On Set-Valued Stochastic Integrals,” Stochastic Analysis and Applications, Vol. 21, No. 2, 2003, pp. 401-408. http://dx.doi.org/10.1081/SAP-120019292

[5] S. Li and A. Ren, "Representation Theorems, Set-Valued and Fuzzy Set-Valued Ito Integral,” Fuzzy Sets and Systems, Vol. 158, No. 9, 2007, pp. 949-962. http://dx.doi.org/10.1016/j.fss.2006.12.004

[6] J. Zhang, "Set-Valued Stochastic Integrals with Respect to a Real Valued Maringale," Soft Method for Handling Vaelability and Imprecision ASC 48, Spinger-Verlag, Berlin Herdelberg, 2008.

[7] J. Zhang, S. Li, I. Mitoma and Y. Okazaki, "On Set-Valued Stochastic Integrals in M-Type 2 Banach Space,”
Journal of Mathematical Analysis and Applications, Vol. 350, No. 1, 2009, pp. 216-233.

http://dx.doi.org/10.1016/j.jmaa.2008.09.017

[8] J. Zhang, S. Li, I. Mitoma and Y. Okazaki, "On the Solution of Set-Valued Stochastic Differential Equations in M-Type 2 Banach Space,” Tohoku Mathematical Journal, Vol. 61, No. 3, 2009, pp. 417-440. http://dx.doi.org/10.2748/tmj/1255700202

[9] J. Zhang, "Integrals and Stochastic Differential Equations for Set-Valued Stochastic Processes,” Ph.D. Thesis, Saga University, Saga, 2009.

[10] I. Mitoma, Y. Okazaki and J. Zhang, "Set-Valued Stochastic Differential Equations in M-Type 2 Banach Space," Communications on Stochastic Analysis, Vol. 4, No. 2, 2010, pp. 215-237.

[11] J. G. Li, S. Li and Y. Ogura, "Strong Solutions of Ito Type Set-Valued Stochastic Differential Equation,” Acta Mathematica Sinica, English Series, Vol. 26, No. 9, 2010, pp. 1739-1748.

http://dx.doi.org/10.1007/s10114-010-8298-x

[12] M. Malinowski and M. Michta, "Set-Valued Stochastic Integral Equations Driven by Martingales," Journal of Mathematical Analysis and Applications, Vol. 394, No. 12, 2012, pp. 30-47. http://dx.doi.org/10.1016/j.jmaa.2012.04.042

[13] J. Zhang, I. Mitoma and Y. Okazaki, "Set-Valued Stochastic Integrals with Respect to Poisson Processes in a Banach Space," International Journal of Approximate Reasoning, Vol. 54, No. 3, 2013, pp. 404-417. http://dx.doi.org/10.1016/j.ijar.2012.06.001

[14] Z. Wang and R. Wang, "Set-Valued Lebesgue-Stieltjes Integrals,” Journal of Applied Probability and Statistics, Vol. 13, No. 3, 1997, pp. 303-316.

[15] S. Li, Y. Ogura and Y. Kreinovich, "Limit Theorems and Applications of Set-Valued and Fuzzy Set-Valued Random Variables," 43rd Edition, Kluwer Academic Publishers, Dordrecht, 2002. http://dx.doi.org/10.1007/978-94-015-9932-0

[16] W. Zhang, S. Li, Z. Wang and Y. Gao, “An Introduction about Set-Valued Stochastic Process,” Science Press, Beijing, 2007.

[17] L. Wang and H. Xue, "Set-Valued Lebesgue-Stieltjes Integrals,” Basic Sciences Journal of Textile Universities, Vol. 16, No. 4, 2004, pp. 317-320. 